\title{
SYSTEM REPRESENTATION AND OPTIMAL TRACKING IN DATA SPACE
}

\author{
Yasumasa Fujisaki* Yiran Duan*, and \\ Masao Ikeda ${ }^{* *}$ \\ * Kobe University, Kobe 657-8501, Japan \\ ** Osaka University, Suita, Osaka 565-0871, Japan
}

\begin{abstract}
Optimal tracking is considered for a linear time-invariant plant in data space. The proposed control strategy does not employ any traditional mathematical model such as a transfer function or a state-space equation. Instead, the plant dynamics is represented as a set of basis vectors whose elements are inputoutput data of the plant. Using this system representation, an optimal tracking problem is solved, which is to find the control input which minimizes a quadratic performance index subject to achieving dead-beat tracking for arbitrary reference signals. Copyright (C) 2005 IFAC
\end{abstract}

Keywords: System theory, Data sets, Optimal control, Tracking system.

\section{INTRODUCTION}

Data driven control provides a simple control strategy based on observed time-series generated by a plant. In fact, we do not need any traditional mathematical model of the plant such as a transfer function, a state equation (Kalman, 1960), or a kernel representation (Willems, 1991) if available information about the plant is its input-output data. The data themselves represent the plant dynamics. Thus, based on a sufficient number of the data, we can control the plant, without introducing any traditional mathematical model.

Several independent ideas regarding data driven control have been proposed in the context of optimal control. See, for example, Chan (1996), Furuta and Wongsaisuwan (1995), Kawamura (1998), and Skelton and Shi (1994), where a mathematical model (e.g., a state equation or a difference equation) of the optimal controller is derived directly form the observed data. However, these papers focus on algorithms to find the controller, and dynamical system theory for data driven control is still underdeveloped.
In order to develop a comprehensive framework for data driven control, the authors have proposed a dynamical system theory in data space (Fujisaki, Duan, and Ikeda, 2004), which is based on the idea proposed by Ikeda, Fujisaki, and Hayashi (2001). This approach employs a system representation in data space instead of a transfer function or a state equation, and it provides a self-contained theory for data driven control. In fact, this approach requires neither the mathematical model of the plant nor that of the controller. In this sense, this approach realizes a purified version of the behavioral approach (Willems, 1991), i.e., the plant is described by its behavior and the control input is also derived by the behavior.

In the framework provided by Fujisaki et al. (2004), the plant dynamics is represented as a set of basis vectors whose elements are inputoutput data of the plant, which determines the data space of the plant. The structure of this space is investigated, where several subspaces, e.g., reachable data space and controllable data space are introduced. Based on these discussions, two optimal regulation problems are solved in 
data space. One is to find the control input which minimizes a quadratic performance index. The other is to find the control input which minimizes a quadratic performance index subject to achieving dead-beat regulation.

The present paper follows this line of research. The objective of this paper is to develop a control strategy in data space which achieves an optimal tracking for linear discrete time plant. The control problem is to find the control input which minimizes a quadratic performance index subject to achieving dead-beat tracking for a given reference signal. Unlike the previous paper (Fujisaki et al., 2004) regarding regulation, tracking problem requires a different subspace in data space, which is investigated extensively in this paper. The result suggests that we need suitable subspaces in data space in order to solve a particular control problem.

The outline of this paper is as follows. In Section 2, we give a system representation in data space, and investigate its subspaces which is suitable for dead-beat tracking. In Section 3, based on this structure of the data space, we derive a way to solve an optimal dead-beat tracking problem in data space. Algorithms for computation of bases of the subspaces are also provided. Section 4 presents a numerical example. In Section 5 , we make some concluding remarks. The detailed proofs may be found in Appendix A

\section{SYSTEM REPRESENTATION IN DATA SPACE}

In this paper, we consider a finite dimensional, linear, discrete time, shift invariant plant with single input and single output. This system can be represented as a difference equation

$$
\sum_{i=0}^{n} \alpha_{i} y_{k-i}=\sum_{i=r}^{n} \beta_{i} u_{k-i}
$$

where $u_{k} \in \mathcal{R}$ and $y_{k} \in \mathcal{R}$ are the input and the output at time $k$ respectively. Furthermore, $n$ and $r$ denote the MacMillan degree and the relative degree of the plant, and $n \geq r$. The parameters $\alpha_{i} \in \mathcal{R}$ and $\beta_{i} \in \mathcal{R}$ are constant which satisfy $\alpha_{0} \neq 0$ and $\beta_{r} \neq 0$.

We suppose that the transfer function of the plant (1) is coprime, and $n$ and $r$ are known. On the other hand, we do not assume that $\alpha_{i}$ and $\beta_{i}$ are known. Instead, we assume that a sufficient number of input-output data $y_{k}, u_{k}$ generated by the plant are available. Then, we present a system representation and a control strategy based on the data themselves. Note that we need $r$ in order to compute an input to achieve dead-beat tracking for arbitrary reference signals even if we take transfer function approach or state space approach.

Let us first introduce a data vector which consists of $\ell$ step outputs and $\ell-r$ step inputs from time $k$, i.e.,

$$
\begin{aligned}
& z_{r}=\left[\begin{array}{llll}
y_{k} & y_{k+1} & \cdots & y_{k+\ell-1}
\end{array},\right. \\
& \left.\begin{array}{llll}
u_{k} & u_{k+1} & \cdots & u_{k+\ell-r-1}
\end{array}\right]^{\mathrm{T}}
\end{aligned}
$$

where $\ell>n$. We use the inputs not till $k+\ell-1$ but till $k+\ell-r-1$ because the inputs from $k+\ell-r$ do not affect the outputs till $k+\ell-1$.

All admissible data vectors are constrained by the difference equation (1), thus $z_{r}$ generated by the plant belongs to a subspace in the vector space $\mathcal{R}^{2 \ell-r}$. We call this subspace the data space, which is denoted by $\mathcal{Z}_{r}$. Here we state the following theorem.

\section{Theorem 1.}

$$
\operatorname{dim}\left(\mathcal{Z}_{r}\right)=\ell+n-r
$$

That is, any data vector $z_{r}$ can be represented as a linear combination of $\ell+n-r$ basis vectors of $\mathcal{Z}_{r}$. We therefore regard a basis of $\mathcal{Z}_{r}$ as a system representation of the plant, which is itself a set of admissible data vectors. In the rest of this section, we investigate structures of $\mathcal{Z}_{r}$, which will be used for dead-beat tracking.

Let us define the initial series of the data vector (2) as its inputs and outputs in the first $n$ steps. We consider a data vector whose initial series is 0 , i.e.,

$$
\begin{aligned}
& z_{r F}=\left[\begin{array}{llllll}
0 & \cdots & 0 & y_{k+n} & \cdots & y_{k+\ell-1}{ }^{\prime}
\end{array}\right. \\
& \left.\begin{array}{llllll}
0 & \cdots & 0 & u_{k+n} & \cdots & u_{k+\ell-r-1}
\end{array}\right]^{\mathrm{T}}
\end{aligned}
$$

where $\ell>n+r$. We call the set of all $z_{r F}$ generated by the plant the reachable data space, which is denoted by $\mathcal{Z}_{r F}$. Then, we obtain the following theorem.

Theorem 2.

$$
\operatorname{dim}\left(\mathcal{Z}_{r F}\right)=\ell-n-r
$$

From Theorems 1 and 2, we see that the data space $\mathcal{Z}_{r}$ can be represented as a direct sum

$$
\mathcal{Z}_{r}=\mathcal{Z}_{r I} \oplus \mathcal{Z}_{r F}
$$

where

$$
\operatorname{dim}\left(\mathcal{Z}_{r I}\right)=2 n \text {. }
$$

That is, any data vector $z_{r}$ has a unique decomposition

$$
z_{r}=z_{r I}+z_{r F}
$$

where $z_{r I} \in \mathcal{Z}_{r I}$ whose initial series is identical to that of $z_{r}$, and $z_{r F} \in \mathcal{Z}_{r F}$.

Let us define the output terminal series of the data vector (2) as its outputs in the last $s$ steps, where 
$s \in \mathbb{N}$. The output terminal series represents an arbitrary reference signal in the context of deadbeat tracking, and its length $s$ corresponds to the time interval such that the output of the plant is required to be identical to the reference signal.

Remark 3. In this paper, we introduce the output terminal series of (2) as its outputs in the last $s$ steps, which will be used for a dead-beat tracking for arbitrary reference signals. On the other hand, in the authors' previous work (Fujisaki et al., $2004)$, the terminal series of (2) is defined as its inputs and outputs in the last $n$ steps, and a deadbeat regulation is considered for the steady state.

Now, we consider a data vector whose output terminal series is 0, i.e.,

$$
\begin{aligned}
z_{r P y}=\left[\begin{array}{llllll}
y_{k} & \cdots & y_{k+\ell-s-1} & 0 & \cdots & 0_{1}^{\prime} \\
u_{k} & u_{k+1} & \cdots & u_{k+\ell-r-1}
\end{array}\right]^{\mathrm{T}}
\end{aligned}
$$

where $\ell>s$. We call the set of all $z_{r P y}$ generated by the plant the output controllable data space, which is denoted by $\mathcal{Z}_{r P y}$. Then, we have the following theorem.

\section{Theorem 4.}

$$
\operatorname{dim}\left(\mathcal{Z}_{r P y}\right)=\ell+n-r-s
$$

From Theorems 1 and 4, we see that the data space $\mathcal{Z}_{r}$ can be represented as a direct sum

$$
\mathcal{Z}_{r}=\mathcal{Z}_{r T y} \oplus \mathcal{Z}_{r P y}
$$

where

$$
\operatorname{dim}\left(\mathcal{Z}_{r T y}\right)=s .
$$

That is, any data vector $z_{r}$ has also a unique decomposition

$$
z_{r}=z_{r T y}+z_{r P y}
$$

where $z_{r T y} \in \mathcal{Z}_{r T y}$ whose output terminal series is identical to that of $z_{r}$, and $z_{r P y} \in \mathcal{Z}_{r P y}$.

Notice here that dead-beat tracking for arbitrary reference signals can be recast as a problem: Find a data vector which has a given initial series corresponding to the initial state of the plant and a specified output terminal series corresponding to the reference signal. In this context, $\mathcal{Z}_{r}$ should contain all data vectors having arbitrary initial series and arbitrary output terminal series. A necessary condition to meet this requirement is

$$
\operatorname{dim}\left(\mathcal{Z}_{r}\right) \geq \operatorname{dim}\left(\mathcal{Z}_{r I}\right)+\operatorname{dim}\left(\mathcal{Z}_{r T y}\right)
$$

that is, $\ell \geq n+r+s$. Under the assumption $\ell \geq n+r+s$, we obtain the following theorem.

\section{Theorem 5.}

$$
\operatorname{dim}\left(\mathcal{Z}_{r P y} \cap \mathcal{Z}_{r F}\right)=\ell-n-r-s .
$$

Thus, under the condition $\ell \geq n+r+s$,

$$
\begin{aligned}
& \operatorname{dim}\left(\mathcal{Z}_{r P y}+\mathcal{Z}_{r F}\right) \\
& =\operatorname{dim}\left(\mathcal{Z}_{r P y}\right)+\operatorname{dim}\left(\mathcal{Z}_{r F}\right)-\operatorname{dim}\left(\mathcal{Z}_{r P y} \cap \mathcal{Z}_{r F}\right) \\
& =\operatorname{dim}\left(\mathcal{Z}_{r}\right)
\end{aligned}
$$

holds. This means that

$$
\mathcal{Z}_{r}=\mathcal{Z}_{r P y}+\mathcal{Z}_{r F} .
$$

We rewrite the data space $\mathcal{Z}_{r}$ with $\ell \geq n+r+s$ as a direct sum

$$
\mathcal{Z}_{r}=\mathcal{Z}_{r I P y} \oplus \mathcal{Z}_{r T y F} \oplus \mathcal{Z}_{r C y}
$$

where

$$
\mathcal{Z}_{r C y}=\mathcal{Z}_{r P y} \cap \mathcal{Z}_{r F} .
$$

Here, from Theorems 2, 4, and 5, the subspaces $\mathcal{Z}_{r T y F} \subseteq \mathcal{Z}_{r F}$ and $\mathcal{Z}_{r I P y} \subseteq \mathcal{Z}_{r P y}$ satisfy

$$
\operatorname{dim}\left(\mathcal{Z}_{r I P y}\right)=2 n, \quad \operatorname{dim}\left(\mathcal{Z}_{r T y F}\right)=s .
$$

The relation (11) means that any data vector $z_{r}$ has a unique decomposition

$$
z_{r}=z_{r I P y}+z_{r T y F}+z_{r C y}
$$

where $z_{r I P y} \in \mathcal{Z}_{r I P y}$ whose initial series is identical to that of $z_{r}$ and whose output terminal series is $0, z_{r T y F} \in \mathcal{Z}_{r T y F}$ whose initial series is 0 and whose output terminal series is identical to that of $z_{r}$, and $z_{r C y} \in \mathcal{Z}_{r C y}$ whose initial and output terminal series are both 0 . Utilizing this fact, as we will see in Section 3, we can solve an optimal dead-beat tracking problem based on the system representation in data space.

\section{OPTIMAL TRACKING IN DATA SPACE}

In this section, based on the structures of the data space, we consider optimal dead-beat tracking for arbitrary reference signals as an optimal control with finite horizon. We use a performance index

$$
J_{r}=\left(z_{r R}-z_{r}\right)^{\mathrm{T}} Q_{r}\left(z_{r R}-z_{r}\right)
$$

where $z_{r} \in \mathcal{Z}_{r}$ is a data vector of the plant, $z_{r R} \in \mathcal{R}^{2 \ell-r}$ is a given reference data vector, and $Q_{r} \in \mathcal{R}^{(2 \ell-r) \times(2 \ell-r)}$ is a given positive definite matrix.

Suppose that $\ell \geq n+r+s$. Suppose also that we start controlling the plant at time $k+n$, which implies that the data until this time are known and the initial series of $z_{r}$ is specified. Our objective is to find an input series to achieve a dead-beat tracking for a given arbitrary reference signal, i.e., control inputs such that the output is identical to the reference signal from $k+\ell-$ $s$ till $k+\ell-1$. As we have seen in Section 2, $z_{r I P y} \in \mathcal{Z}_{r I P y}$ whose initial series is identical to that of $z_{r}$ and whose output terminal series is 0 and $z_{r T y F} \in \mathcal{Z}_{r T y F}$ whose initial series is 0 and whose output terminal series is identical to that of $z_{r}$ are uniquely determined. Thus, optimal dead-beat tracking control problem is formulated as follows. 
Problem 6. For given $z_{r R} \in \mathcal{R}^{2 \ell-r}, \hat{z}_{r I P y} \in$ $\mathcal{Z}_{r I P y}$, and $\hat{z}_{r T y F} \in \mathcal{Z}_{r T y F}$, find the optimal data vector $z_{\text {ropt }} \in \mathcal{Z}_{r}$ which minimizes the performance index $J_{r}$ of (12) subject to

$$
z_{r}=\hat{z}_{r I P y}+\hat{z}_{r T y F}+z_{r C y}
$$

where $z_{r C y} \in \mathcal{Z}_{r C y}$ is the decision variable.

Since the quadratic form $x^{\mathrm{T}} Q_{r} x$ can be regarded as a metric in the inner product space $\mathcal{R}^{2 \ell-r}$, the optimal data vector $z_{r C y o p t} \in \mathcal{Z}_{r C y}$ which minimizes $J_{r}$ of (12) can be represented as

$$
z_{r C y o p t}=P_{r C y}\left(z_{r R}-\hat{z}_{r I P y}-\hat{z}_{r T y F}\right)
$$

where $P_{r C y}$ is the $Q_{r}$-orthogonal projection onto $\mathcal{Z}_{r C y}$ in $\mathcal{R}^{2 \ell-r}$. It is given by

$$
P_{r C y}=H_{r C y}\left(H_{r C y}^{\mathrm{T}} Q_{r} H_{r C y}\right)^{-1} H_{r C y}^{\mathrm{T}} Q_{r}
$$

where $H_{r C y}$ is a matrix whose columns consist of a basis of $\mathcal{Z}_{r C y}$. Then, we obtain the following theorem.

Theorem 7. For given $z_{r R} \in \mathcal{R}^{2 \ell-r}, \hat{z}_{r I P y} \in$ $\mathcal{Z}_{r I P y}$, and $\hat{z}_{r T y F} \in \mathcal{Z}_{r T y F}$, there exists a unique $z_{\text {ropt }} \in \mathcal{Z}_{r}$ which minimizes $J_{r}$ of (12) subject to (13), and it is given by

$$
z_{r o p t}=\left(I-P_{r C y}\right)\left(\hat{z}_{r I P y}+\hat{z}_{r T y F}\right)+P_{r C y} z_{r R} .
$$

In this way, we obtain $z_{\text {ropt }}$. The elements of $z_{\text {ropt }}$ corresponding to $u_{k+n}, u_{k+n+1}, \ldots, u_{k+\ell-r-1}$ are the optimal inputs to be applied at times $k+n$, $k+n+1, \ldots, k+\ell-r-1$.

In order to compute $z_{\text {ropt }}$, we need bases of the subspaces $\mathcal{Z}_{r I P y}, \mathcal{Z}_{r T y F}$, and $\mathcal{Z}_{r C y}$. To this end, let us introduce a block Hankel matrix of $y_{i}$ and $u_{i}$

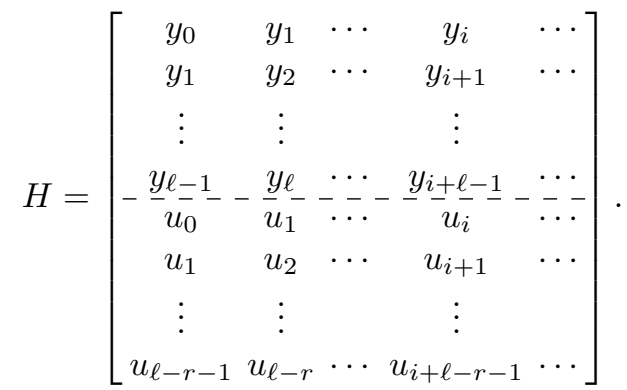

When a sufficient number of data are available, Theorem 1 ensures that $H$ contains $\ell+n-r$ independent column vectors. We denote $H_{Z}$ as a matrix whose columns consist of these vectors. Then, we have the following theorem.

Theorem 8. A column-equivalent matrix of $H_{Z}$ given by elementary column operations is represented as

$$
\left[\begin{array}{lll}
H_{r I P y} & H_{r T y F} & H_{r C y}
\end{array}\right]=\left[\begin{array}{cc|c|c}
I_{n} & 0 & 0 & 0 \\
* & * & * & * \\
0 & 0 & I_{s} & 0 \\
\hdashline 0 & \bar{I}_{n} & 0 & 0 \\
* & * & * & *
\end{array}\right]
$$

where $H_{r I P y}, H_{r T y F}$, and $H_{r C y}$ are bases of $\mathcal{Z}_{r I P y}, \mathcal{Z}_{r T y F}$, and $\mathcal{Z}_{r C y}$, respectively. Here * represents appropriate matrices determined by the operations.

From this theorem, we can construct $P_{r C y}$. Furthermore, we also construct $\hat{z}_{r I P y} \in \mathcal{Z}_{r I P y}$ and $\hat{z}_{r T y F} \in \mathcal{Z}_{r T y F}$ using the bases given in the above. To show this fact, let us define an initial series vector as

$$
\begin{aligned}
& x_{I}=\left[\begin{array}{llll}
y_{k} & y_{k+1} & \cdots & y_{k+n-1}
\end{array},\right. \\
& \left.\begin{array}{llll}
u_{k} & u_{k+1} & \cdots & u_{k+n-1}
\end{array}\right]^{\mathrm{T}}
\end{aligned}
$$

which consists of the data obtained by the initial time $k+n$. Then, $\hat{z}_{r I P y} \in \mathcal{Z}_{r I P y}$ whose initial series is identical to $x_{I}$ is given by

$$
\hat{z}_{r I P y}=H_{r I P y} x_{I} .
$$

Similarly, we define an output terminal series vector as

$$
x_{T y}=\left[\begin{array}{llll}
y_{k+\ell-s} & y_{k+\ell-s+1} & \cdots & y_{k+\ell-1}
\end{array}\right]^{\mathrm{T}}
$$

which consists of the given reference signal. Then, $\hat{z}_{r T y F} \in \mathcal{Z}_{r T y F}$ whose output terminal series is identical to $x_{T y}$ is given by

$$
\hat{z}_{r T y F}=H_{r T y F} x_{T y} .
$$

In this way, we can determine $P_{r C y}, \hat{z}_{r I P y} \in$ $\mathcal{Z}_{r I P y}$, and $\hat{z}_{r T y F} \in \mathcal{Z}_{r T y F}$. Using these matrix and vectors together with the reference data vector $z_{r R}$, we can compute $z_{\text {ropt }}$ following Theorem 7 .

Remark 9. If $\ell=n+r+s$, then $\mathcal{Z}_{r C y}=\emptyset$. In this case, $z_{r}$ of (13) is uniquely determined, and the minimum step dead-beat tracking is achieved. In our context, the algorithm proposed by Ikeda et al. (2001) can be expressed like the above. They set $s=1$, i.e., $\ell=n+r+1$, and compute the input in a sequential manner. Obviously, if we consider this kind of control with infinite horizon, the plant should be of minimum phase in order to obtain a bounded input series (Ikeda et al., 2001).

\section{NUMERICAL EXAMPLE}

In this section, we summarize the procedure proposed in the previous section through a numerical example. We consider a plant with the MacMillan degree $n=2$ and the relative degree $r=1$ of the form

$$
y_{k}+0.7 y_{k-1}+0.1 y_{k-2}=u_{k-1}-0.5 u_{k-2} .
$$

We here assume that the parameters of the plant are unknown but a sufficient number of the inputoutput data generated by the plant are known. Then, we demonstrate that the procedure gives the optimal dead-beat tracking input directly 
from the data. In this example, we set $\ell=7$ and $s=2$.

Suppose that an input series to the plant

$$
\begin{array}{lll}
u_{0}=-1, & u_{1}=1, & u_{2}=-1, \\
u_{3}=1, & u_{4}=1, & u_{5}=-1, \\
u_{6}=-1, & u_{7}=1, & u_{8}=-1, \\
u_{9}=-1, & u_{10}=-1, & u_{11}=1, \\
u_{12}=1, & u_{13}=1, & u_{14}=1
\end{array}
$$

and the corresponding output series

$$
\begin{array}{lll}
y_{0}=0, & y_{1}=-1.00, & y_{2}=2.20 \\
y_{3}=-2.94, & y_{4}=3.34, & y_{5}=-1.54 \\
y_{6}=-0.75, & y_{7}=0.18, & y_{8}=1.45 \\
y_{9}=-2.53, & y_{10}=1.13, & y_{11}=-1.04 \\
y_{12}=2.11, & y_{13}=-0.88, & y_{14}=0.90
\end{array}
$$

are observed. We substitute the output data till time 14 and the input data till time 13 into $H$ of (15) with $\ell=7$. Then, $H$ becomes $13 \times 8$ matrix having full column rank, thus it can be regarded as $H_{Z}$. Following Theorem 8, we obtain a columnequivalent matrix of $H_{Z}$ as

$$
\begin{aligned}
& {\left[\begin{array}{lll}
H_{r I P y} & H_{r T y F} & H_{r C y}
\end{array}\right]} \\
& =\left[\begin{array}{rrrr|rr|rr}
1 & 0 & 0 & 0 & 0 & 0 & 0 & 0 \\
0 & 1 & 0 & 0 & 0 & 0 & 0 & 0 \\
-0.10 & -0.70 & -0.50 & 1.00 & 0 & 0 & 0 & 0 \\
0 & 0 & 0 & 0 & 0 & 0 & 1 & 0 \\
0 & 0 & 0 & 0 & 0 & 0 & 0 & 1 \\
0 & 0 & 0 & 0 & 1 & 0 & 0 & 0 \\
0 & 0 & 0 & 0 & 0 & 1 & 0 & 0 \\
---5---0---\frac{1}{1}- & -0 & - & 0 & 0 & -\frac{1}{0}- & - \\
0 & 0 & 0 & 1 & 0 & 0 & 0 & 0 \\
-0.07 & -0.39 & -0.35 & 1.20 & 0 & 0 & 1 & 0 \\
-0.05 & -0.27 & -0.22 & 0.70 & 0 & 0 & 1.20 & 1 \\
-0.02 & -0.13 & -0.11 & 0.35 & 1 & 0 & 0.70 & 1.20 \\
-0.01 & -0.07 & -0.06 & 0.17 & 1.20 & 1 & 0.35 & 0.70
\end{array}\right]
\end{aligned}
$$

When we choose $Q_{r}$ in the performance index as

$$
Q_{r}=\text { block } \operatorname{diag}\left\{I_{\ell}, 2 I_{\ell-r}\right\}
$$

we can compute the $Q_{r}$-orthogonal projection $P_{r C y}$ using (14), which is given in Appendix B.

We choose the reference data vector as

$$
z_{r R}=\left[\begin{array}{llllllllll}
-1 & -0.5 & 0 & 0.5 & 1 & 0.5 & 0 & 0 & \cdots & 0
\end{array}\right]^{\mathrm{T}} .
$$

That is, we consider the control problem such that the output tracks a triangular wave. Since the data till time 14 are obtained, we use the data at times 13 and 14 as the initial series and start the optimal tracking at time 15, i.e.,

$$
x_{I}=\left[\begin{array}{llll}
-0.88 & 0.90^{\prime} & 1 & 1
\end{array}\right]^{\mathrm{T}} .
$$

Furthermore, since $\ell=7$ and $s=2$, the output terminal series vector can be taken form $z_{r R}$ as

$$
x_{T y}=\left[\begin{array}{ll}
0.5 & 0
\end{array}\right]^{\mathrm{T}} .
$$

Using the above, from Theorem 7 , we obtain the solution $z_{\text {ropt }}$ of the optimal dead-beat tracking as

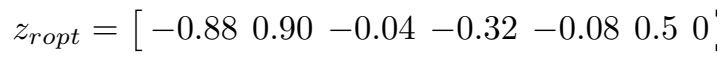

$$
\begin{aligned}
& \left.\begin{array}{llllll}
1 & 1 & 0.24 & -0.19 & 0.32 & 0.50
\end{array}\right]^{\mathrm{T}} .
\end{aligned}
$$

That is, the optimal inputs are given by

$$
\begin{aligned}
& u_{15}=0.24, \quad u_{16}=-0.19, \quad u_{17}=0.32, \\
& u_{18}=0.50 .
\end{aligned}
$$

\section{CONCLUDING REMARKS}

In this paper, we have studied an optimal deadbeat tracking for arbitrary reference signals based on a system representation in data space. We have demonstrated that this control problem can be actually solved directly from the observed data, without employing any traditional mathematical model such as a transfer function or a state equation.

Note that we have assumed that both of the MacMillan degree and the relative degree of the plant are known. Then, we have introduced subspaces of the data space which are consistent with the a priori information. These subspaces were useful for realization of the optimal dead-beat tracking such that the output follows the given reference signal without tracking error in a finite number of steps. On the other hand, Fujisaki et al. (2004) assume that only the MacMillan degree of the plant is known. Then, different subspaces of the data space are provided and a dead-beat regulation is solved such that both of the input and the output reach a steady state in a finite number of steps. These results suggest that we may solve a particular control problem if we introduce suitable subspaces of the data space which are consistent with a priori information.

\section{REFERENCES}

Chan, J. T. H. (1996). Data based Synthesis of a Multivariable Linear Quadratic Regulator. Automatica, 32(3), 403-407.

Fujisaki, Y., Y. Duan, and M. Ikeda (2004). System Representation and Optimal Control in Input-Output Data Space. Preprints of the 10th IFAC/IFORS/IMACS/IFIP Symposium on Large Scale Systems: Theory and Applications, 197-202.

Furuta, K. and M. Wongsaisuwan (1995). Discrete-time LQG Dynamic Controller Design Using Plant Markov Parameters. Automatica, 31(9), 1317-1324.

Ikeda, M., Y. Fujisaki, and N. Hayashi (2001). A Model-less Algorithm for Tracking Control Based on Input-Output Data. Nonlinear 
Analysis: Theory, Methods \& Applications, 47(3), 1953-1960.

Kalman, R. E. (1960). On the General Theory of Control Systems. Proceedings of the first World Congress of IFAC, 481-492.

Kawamura, Y. (1998). Direct Construction of LQ Regulator Based on Orthogonalization of Signals: Dynamical Output Feedback. Systems $\&$ Control Letters, 34, 1-9.

Skelton, R. E. and G. Shi (1994). The DataBased LQG Control Problem. Proceedings of the 33rd IEEE Conference on Decision and Control, 1447-1452.

Willems, J. C. (1991). Paradigms and Puzzles in the Theory of Dynamical Systems. IEEE Transactions on Automatic Control, 36(3), 259-294.

\section{Appendix A. PROOFS OF THE THEOREMS}

We first give the proofs of Theorems $1,2,4$, and 5 . Note that $\ell>n$. We define $(\ell-n) \times(2 \ell-r)$ matrix which contains the coefficients of the difference equation (1) as

$$
\Theta_{r}=\left[\begin{array}{rrrrrrr}
\alpha_{n} & \alpha_{n-1} & \cdots & \alpha_{0} & & 0 & \\
& \ddots & \ddots & & \ddots & & \\
0 & & \alpha_{n} & \alpha_{n-1} & \cdots & \alpha_{0} & \\
-\beta_{n}-\beta_{n-1} & \cdots & -\beta_{r} & & 0 \\
& \ddots & \ddots & & \ddots & \\
0 & & -\beta_{n} & -\beta_{n-1} & \cdots & -\beta_{r}
\end{array}\right] .
$$

Then, we see that, for any data vector $z_{r}$,

$$
\Theta_{r} z_{r}=0
$$

holds. Since we assume that the coprimeness of the transfer function of the plant (1), the relation (A.1) represents the constraint on data vectors $z_{r}$. We therefore see that

$$
\mathcal{Z}_{r}=\operatorname{Ker} \Theta_{r}
$$

We further consider the other subspaces of $\mathcal{Z}_{r}$. Suppose that $\ell$ is appropriate. We define

$$
\begin{aligned}
J_{r F} & =\operatorname{block} \operatorname{diag}\left\{I_{n}, 0_{\ell-n}, I_{n}, 0_{\ell-n-r}\right\} \\
J_{r P y} & =\text { block } \operatorname{diag}\left\{0_{\ell-s}, I_{s}, 0_{\ell-r}\right\}
\end{aligned}
$$

which correspond to (4) and (7), respectively. Then, we rewrite the constraints such that the initial series or the output terminal series is 0 as

Thus, we see that

$$
J_{r F} z_{r F}=0, \quad J_{r P y} z_{r P y}=0 .
$$

$$
\begin{aligned}
\mathcal{Z}_{r F} & =\operatorname{Ker} \Theta_{r} \cap \operatorname{Ker} J_{r F} \\
\mathcal{Z}_{r P y} & =\operatorname{Ker} \Theta_{r} \cap \operatorname{Ker} J_{r P y} \\
\mathcal{Z}_{r C y} & =\operatorname{Ker} \Theta_{r} \cap \operatorname{Ker} J_{r P y} \cap \operatorname{Ker} J_{r F} .
\end{aligned}
$$

Notice that $\alpha_{0} \neq 0$ and $\beta_{r} \neq 0$. It turns out that the ranks of the matrices

$$
\Theta_{r}, \quad\left[\begin{array}{c}
\Theta_{r} \\
J_{r F}
\end{array}\right], \quad\left[\begin{array}{c}
\Theta_{r} \\
J_{r P y}
\end{array}\right], \quad\left[\begin{array}{c}
\Theta_{r} \\
J_{r F} \\
J_{r P y}
\end{array}\right]
$$

are $\ell-n, \ell+n, \ell-n+s$, and $\ell+n+s$, respectively. We therefore see that

$$
\begin{aligned}
\operatorname{dim}\left(\mathcal{Z}_{r}\right) & =\operatorname{dim}\left(\operatorname{Ker} \Theta_{r}\right) \\
& =2 \ell-r-\operatorname{rank} \Theta_{r} \\
& =\ell+n-r \\
\operatorname{dim}\left(\mathcal{Z}_{r F}\right) & =\ell-n-r \\
\operatorname{dim}\left(\mathcal{Z}_{r P y}\right) & =\ell+n-r-s \\
\operatorname{dim}\left(\mathcal{Z}_{r C y}\right) & =\ell-n-r-s
\end{aligned}
$$

which give the results of Theorems $1,2,4$, and 5 .

We next give the proof of Theorem 8. Permuting the rows of $H_{Z} \in \mathcal{R}^{(2 \ell-r) \times(\ell+n-r)}$ appropriately, we have $\widehat{H}_{Z}$ whose first $2 n+s$ rows correspond to the initial series and the output terminal series. Theorem 5 says that the dimension of $\mathcal{Z}_{r C y}$ is $\ell-n-r-s$. Thus, executing elementary column operations on $\widehat{H}_{Z}$, we have

$$
\left[\begin{array}{cc}
\widehat{H}_{11} & 0 \\
\widehat{H}_{21} & \widehat{H}_{22}
\end{array}\right] \text {. }
$$

Note that $\widehat{H}_{11} \in \mathcal{R}^{(2 n+s) \times(2 n+s)}$ is a nonsingular matrix, thus it can be identity matrix by elementary column operations. Permuting the rows of $\widehat{H}_{Z}$ again in the converse way, we obtain the matrix (16), which gives the result of Theorem 8 .

\section{Appendix B. ORTHOGONAL PROJECTION}

The $Q_{r}$-orthogonal projection $P_{r C y}$ in the numerical example is given by

$$
P_{r C y}=\left[\begin{array}{rrrrrrrr}
0 & 0 & 0 & 0 & 0 & 0 & 0 & \\
0 & 0 & 0 & 0 & 0 & 0 & 0 & \\
0 & 0 & 0 & 0 & 0 & 0 & 0 & \\
0 & 0 & 0 & 0.25 & -0.16 & 0 & 0 & \\
0 & 0 & 0 & -0.16 & 0.26 & 0 & 0 & \\
0 & 0 & 0 & 0 & 0 & 0 & 0 & \\
0 & 0 & 0 & 0 & 0 & 0 & 0 & \\
0 & 0 & 0 & 0 & 0 & 0 & 0 & \\
0 & 0 & 0 & 0 & 0 & 0 & 0 & \\
0 & 0 & 0 & 0.25 & -0.16 & 0 & 0 & \\
0 & 0 & 0 & 0.13 & 0.06 & 0 & 0 & \\
0 & 0 & 0 & -0.02 & 0.19 & 0 & 0 & \\
0 & 0 & 0 & -0.03 & 0.12 & 0 & 0 & \\
& 0 & 0 & 0 & 0 & & 0 & \\
& 0 & 0 & 0 & 0 & & 0 & 0 \\
& 0 & 0 & 0 & 0 & & 0 & 0 \\
& 0 & 0 & 0.49 & 0.26 & -0.05 & -0.06 \\
& 0 & 0 & -0.33 & 0.12 & 0.38 & 0.24 \\
& 0 & 0 & 0 & 0 & & 0 & 0 \\
& 0 & 0 & 0 & 0 & & 0 & 0 \\
& 0 & 0 & 0 & 0 & & 0 & 0 \\
& 0 & 0 & 0 & 0 & & 0 & 0 \\
& 0 & 0 & 0.49 & 0.26 & -0.05 & -0.06 \\
& 0 & 0 & 0.26 & 0.43 & 0.32 & 0.17 \\
& 0 & 0 & -0.05 & 0.32 & 0.42 & 0.25 \\
& 0 & 0 & -0.06 & 0.17 & 0.25 & 0.15
\end{array}\right]
$$

